\title{
Cloud Service Brokerage: Exploring Characteristics and Benefits of B2B Cloud Marketplaces
}

\author{
Victoria Paulsson, Vincent C. Emeakaroha, \\ John Morrison, and Theo Lynn
}

Abstract With the increasing popularity of cloud computing, a new technology and business model called cloud service brokerage (CSB) is emerging. CSB is, in essence, a middleman in the cloud-computing supply chain to connect prospective cloud buyers with suitable service providers. This chapter focuses on a type of CSB, B2B cloud marketplaces. Recently,

V. Paulsson $(\bowtie)$

Irish Centre for Cloud Computing and Commerce, Dublin, Ireland

V. C. Emeakaroha

Cork Institute of Technology, Cork, Ireland

J. Morrison

University College Cork, Cork, Ireland

T. Lynn

Irish Institute of Digital Business, DCU Business School, Dublin, Ireland

(C) The Author(s) 2020

T. Lynn et al. (eds.), Measuring the Business Value of Cloud

Computing, Palgrave Studies in Digital Business \& Enabling

Technologies, https://doi.org/10.1007/978-3-030-43198-3_4 
this type of marketplace has evolved into two broad categories-business application marketplaces and API marketplaces. This chapter reviews the characteristics of $\mathrm{B} 2 \mathrm{~B}$ cloud marketplaces, and their benefits, which include ease-of-use and ease-of-integration, enhanced security, increased manageability, faster implementation, and cost reduction. The chapter concludes with two mini-case studies, on Salesforce AppExchange and RapidAPI, to illustrate how firms could use B2B cloud marketplaces to generate, capture and measure business value.

Keywords Cloud service brokerage $\bullet$ Application marketplace $\bullet$ Case study • API Marketplace

\subsection{INTRODUCTION}

With the increasing popularity of cloud computing, there is a proliferation of cloud service offerings in the market (Elhabbash et al. 2019). A 2017 report on cloud computing (Columbus 2017) finds that 82 per cent of enterprises are already running projects and applications in the cloud. These businesses are focusing on improving and expanding their use of cloud resources and looking for new ways to gain maximum benefits from the cloud. Attitudes amongst enterprises have improved significantly in the last decade as concerns about cloud security, performance, and available expertise have lessened (Columbus 2017). These positive attitudes toward the cloud in business organisations of various shapes and sizes could result in a higher adoption of business-to-business (B2B) cloud application and API marketplaces (MacInnes 2017).

B2B cloud marketplaces are a type of cloud service brokerage (CSB), an intermediary in the cloud computing value chain. Its main role is to connect prospective cloud users, either individuals or firms, with suitable cloud service providers. While there are CSB definitions available from practitioner literatures, such as Gartner (Lheureux et al. 2012), and MarketsandMarkets (2015), there is no widely accepted definition available in academic literature. This paper subscribes to the definition from the US National Institute of Standards and Technology (NIST) that a CSB is "an entity that manages the use, performance, and delivery of cloud services, and negotiates relationships between Cloud Providers and Cloud Consumers" (Sill et al. 2013). Accordingly, in this chapter, CSB 
refers to a business model and a technology, whose main purpose is to connect prospective cloud service customers with cloud service providers in the cloud.

This chapter aims to provide some insight in to the structure of the CSB landscape, and explore the functional characteristics and benefits of B2B cloud marketplaces. We illustrate these with two mini-case studies on Salesforce AppExchange and RapidAPI.

\subsection{The Four Tiers of Cloud Service Brokerage}

Before we delve deeper into the topic of B2B cloud marketplaces, it is necessary to build a common understanding of the overall structure of the CSB landscape, and where B2B cloud marketplaces are situated. Fowley et al. (2013) categorise CSB from an architecture platform perspective. They identify three platforms: cloud management (Tier 1), cloud broker (Tier 2) and cloud marketplace (Tier 3) platforms. We extend this categorisation by adding a fourth platform, a cloud marketplace enablement platform (Tier 4), as the industry is expanding in this direction since Fowley et al.'s publication. A brief discussion of the these tiers will be useful in distinguishing cloud marketplaces from other CSB tiers.

Tier 1: Cloud management platform-a cloud architecture platform which offers the complete lifecycle of cloud-related management activities such as the design, deployment and provisioning of cloud infrastructure including advanced monitoring of cloud resource utilisation. An exemplar Tier 1 platform use case is a management platform for heterogeneous distributed data centres. These platforms run virtual infrastructures on top of hardware to build private, public or hybrid cloud solutions, such as OpenNebula and Eucalyptus.

Tier 2: Cloud broker platform - supports value added brokering activities such as aggregation, integration, and customisation. These activities require a specific language to make several applications work together in a uniform manner. There are many examples of CSB that specialize in these three core value generators of cloud broker platforms (Lheureux et al. 2012). First, aggregation CSBs bring together multiple services at scale e.g. single sign-on, unified billing, chargeback and show back. Examples of aggregation CSBs include BlueWolf and CloudNation. Second, integration CSBs focus on making multiple clouds work together in an integrated manner e.g. Dell Boomi and HPE Helion. Lastly, customisation 
CSBs build new features and functions on top of existing cloud applications according to business needs e.g. LTech.

Tier 3: Cloud marketplace platform-here a CSB builds upon a cloud broker platform to provide a marketplace, which brings providers and consumers together. Two key features in the cloud marketplace are (i) service descriptions for core and integrated services, and (ii) trust. Two types of cloud marketplace are present in the market at the moment: (1) Business application marketplaces and (2) Application programming interface (API) marketplaces. Business application marketplaces provide cloud application catalogues to perspective business buyers. Examples from the private sector are Microsoft Azure Marketplace, Salesforce AppExchange, Google Apps Marketplace, and the Amazon Web Service (AWS) Marketplace. Examples from the public sector are the Gov.UK Digital Marketplace from the UK government, and the Federal Risk and Authorisation Management Program (FedRAMP) from the US Government. More recently, APIs have emerged as a fundamental building block in the digital economy, connecting organisations, technologies and data. If data is the new oil, APIs are the new pipelines. An API is a set of functions and procedures allowing the creation of applications that access the features or data of a system, application, or other service. They increasingly play an important role in the interoperability of systems, whether cloud-based or otherwise, both internally and externally, and the exchange of data. APIs are bidirectional-they can provide and consume-and can be public (open) or private. A key characteristic of APIs is their abstraction from systems and infrastructure thus allowing third parties to build applications and services that consume APIs. API marketplaces allow cloud software vendors to buy, sell, and/or exchange APIs. Examples include RapidAPI and Apiculture.

Tier 4: Cloud marketplace enablement platform - a multi-tenant platform with a cloud-enabled business model that assists companies to create their own cloud marketplaces. The platform architecture is developed in such a way that it can be licensed, reused and customised to fit many business contexts. Providers of cloud marketplace enablement platforms typically offer a fully integrated platform with a range of value-added services. An exemplar CSB use case in this tier is Marketplace-as-a-service (MaaS). $\mathrm{MaaS}$ is a business model in which a MaaS-oriented firm develops a general cloud marketplace platform and white labels this platform to businesses interested in offering a cloud marketplace as part of their business (Fischer 2012). MaaS clients do not have to reinvent the wheel on the technological 
side of building and managing cloud marketplace infrastructure, so they can focus on strategic issues around marketplaces. AppDirect is an example of a MaaS vendor.

This paper focuses only on Tier 3, B2B cloud application marketplace platforms. The next section will delve into its characteristics.

\subsection{Characteristics of B2B Cloud Marketplace Platforms}

B2B cloud marketplaces are a form of multisided platform business model, in which two or more parties, for example customers and vendors etc., have a direct interaction with one another through the platform (Brokaw 2014). The platform acts as a middleman between buyers and sellers. It earns a commission and/or revenue sharing for transactions taken place through the platform. The marketplaces aggregate a large selection of cloud services, be it business applications or APIs, from multiple software vendors, and offer an integrated service catalogue to cloud customers (Cantara 2015). Aggregation is the core business value that these marketplaces offer, but they could expand to generate value in other ways e.g. integration and customisation. Customers can search for suitable cloud offerings by either browsing through standard categories suggested by the marketplace operators or searching through service descriptions, which accompany every cloud offering (MarketsandMarkets 2015). A successful application or API marketplace attracts a critical mass of customers and software vendors to build economies of scale (Brokaw 2014). Software vendors benefit from access to a large pool of customers which makes an investment to develop new applications and APIs worthwhile. When development costs are spread thinner among a large pool of customers, profits are more likely to increase. Customers also benefit from accessing a large collection of software applications and APIs that could meet their business requirements (Brokaw 2014).

B2B cloud marketplaces offer a wide range of value-added services to the various marketplace actors. First, B2B cloud marketplaces offer many fundamental supports to both sellers and buyers alike. For software vendors, product planning and development, and sales and marketing are some of the areas that marketplace operators offer an invaluable support. Many B2B cloud marketplace platforms run a partner program with software vendors to help them with the application planning and development side 
e.g. the AWS Partner Network, and the Salesforce AppExchange Partner Success program. These partner programmes promote new and existing software vendors to further fine-tune their offerings to fit customer requirements (Gill et al. 2016). Requirements to participate in these programmes depend on a given marketplace operator's policy. A general rule of thumb is that the higher the sales revenue that a cloud application or API generates, the more support it tends to receive from marketplace operators. These partner programmes are beneficial for all parties involved. The software vendors are more confident that their applications and APIs are compatible with what the market is looking for. Cloud service customers are satisfied with cloud solutions they purchase and, as a result, might expand their cloud application and API use into other areas. Cloud marketplace operators earn more brokerage fees as applications and APIs become more popular, and as marketplaces attract more business transactions. These are all positive reinforcements for a marketplace business cycle. Partner portals in marketplaces typically provide vendors with key data, insights, indicators and reports including sales leads, conversion rates, customer feedback, and service usage (Oracle 2015; AWS 2016; Salesforce 2016).

For cloud customers, using a cloud marketplace provides them with two core value-added services: (1) Single-sign-on (SSO) and (2) Integrated event and billing management. Marketplace operators usually provide customers with an ability to use a single username and password to sign onto multiple applications and APIs (AppDirect 2013). SSO has a number of key benefits for firms and their users. First, SSO is associated with increased productivity and improved security. Users enjoy a single point of access to all applications, APIs and resources that they need to get their work done in one convenient portal; they simply log in once to get access to everything they need. They no longer have to waste time finding and logging into separate applications (Kortright 2018). Second, SSO authentication improves overall security from both a system architecture and a behavioural perspective. Architecturally speaking, the SSO authentication model enhances the overall security because security credentials are accepted and processed only at a specific SSO server; no security credentials are transmitted to other systems (Mecca et al. 2016). Behavioural-wise, maintaining multiple passwords for different access points requires a high level of cognitive effort from users. Therefore, users tend to engage in many poor password practices, for example writing down passwords, repeated passwords, and creating a commonly used password for all access points 
(Kortright 2018). SSO minimises risk from poor password practices. Integrated event and billing management involves marketplace operators providing an integrated portal to monitor and manage applications in use. The portal should be sophisticated enough to address and report complex use, billing and monitoring situations such as billing relationships between users, service level and billing intervals, introductory pricing, one-time fees, free trials, upgrades and renewals (Microsoft Azure 2016a; AppDirect 2013). The portal should also allow administrators to view all costs, manage, edit and/or cancel existing subscriptions.

Actors on both sides of the cloud marketplace platforms, software vendors and customers, concurrently weigh the payoffs for conducting business through marketplaces against the external risks inherent in it. Therefore, apart from providing the key value proposition as a cloud application aggregator and value-added service provider, successful cloud marketplace operators must build and maintain their reputation in the following areas to lower perceived external risk levels: (1) financial viability, (2) corporate governance, (3) security and privacy. First, from a financial viability perspective, marketplace vendors should have a solid financial background and a credible reputation in the market. Cloud customers trust the marketplace vendors to manage their access, through SSO, to relevant applications and APIs, as well as business data. A strong financial background provides an assurance that the cloud service will not be interrupted from business discontinuity (Microsoft Azure 2016a). Second, in terms of corporate governance, it is logical to assume that customers are much more likely to conduct a business with cloud marketplace vendors with a strong reputation for corporate governance (Achim et al. 2016). Software vendors and cloud customers, likewise, rely on vendor profiles from reputable sources like Gartner's Magic Quadrant, public ratings and reviews to ensure that their business vision, practices and corporate governance policies are sound (Carraway et al. 2015). Last with regards to security and privacy, vendors should have transparent and compliant data security and privacy policies. Customers should be able to trust that their data and any information processed via the marketplace are secure. Any compromise in this area could seriously undermine the entire viability of cloud marketplace ecosystem since customers on both ends, software vendors and cloud customers, tend to be sensitive to this issue (Sen 2015). Cloud customers are advised to consult service level agreements (SLA) in relation to this matter. Usually marketplace operators operate under a grand-scheme SLA. For example, the FedRAMP Marketplace, 
a cloud marketplace for US federal agencies, ensures that applications listed comply with the US Government's requirements for security in the cloud (DOD 2014). Only applications that have been approved "FedRAMP Ready" are authorised to be listed on the FedRAMP Marketplace (FedRAMP 2019). However, it is possible that specific SLA terms and conditions proffered by certain application vendors may differ from that of the grand-scheme's. Therefore, firms with a strict policy on security and privacy should consult SLAs from both the marketplace operator and vendor to ensure that they are in line with their own policy.

\subsection{Benefits of B2B Cloud Marketplace}

Based on the characteristics of B2B cloud marketplaces explained above, B2B cloud marketplaces offer a number of advantages to customers. First, ease-of-use and ease-of-integration are key advantages that B2B cloud marketplaces offer to cloud customers. As explained earlier, cloud customers can select a cloud application or API that they deem suitable to their business needs, and instantly install it or access it from the cloud. These applications and APIs are typically designed to be intuitive to use or require a bare minimum level of training. Where training is required, software vendors usually provide a wide range of multimedia information, especially video tutorials, to guide users. Users do not have to worry about system upgrades and/or maintenance since software vendors simultaneously and automatically update all underlying software as long as a subscription period is valid (AppDirect 2013). As all cloud marketplace applications and APIs, by definition, are pre-programmed for integration with existing applications, customers can be assured that their newly-acquired applications and APIs will integrate with existing systems (Morin et al. 2012).

Second, enhanced security is an advantage of software licensed through cloud marketplaces. In addition to SSO, cloud marketplace vendors can leverage a world-class level of security provided by a team of security professionals at a fraction of the actual costs incurred. Studies suggest storing data on the cloud is much safer than an on-premise alternative. Key security-related issues such as access control, access authentication, data encryption, firewalls, logs and audit trails are better managed and controlled in the cloud (DPC 2015). These resources are not often within reach for an on-premise data storage solution, simply because of the high cost it entails. 
Increased manageability is the third benefit of sourcing software services through cloud marketplaces. Traditionally, a software license is for a specific version of software for an indefinite period of time in exchange for a specific sum of revenue. Software vendors earned a new wave of revenue by issuing an upgrade. An investment in new software usually involved a large sum of money once other costs are factored in on top of the license cost, for example implementation, customisation and training costs (Malhotra and Majchrzak 2012). Despite rapid changing business environments and shifting customer requirements, many firms find themselves locked-in with outdated software that are no longer suitable for a business purpose, simply because the management cannot justify investing in an upgrade or new software. As the cloud marketplace business model assumes low customer switching costs, software vendors need to ensure customer satisfaction and usage. In comparison to the old licensing model, customers hold an upper hand as they are able to add, remove and modify services whenever they want (Morin et al. 2012). Cloud customers have greater flexibility to manage their software needs as their market and customers' requirements shift, through the integrated event and billing management portal discussed earlier. They can easily scale up, scale down, or switch to a new vendor.

Fourth, faster implementation is another clear benefit from sourcing through a cloud marketplace. Software available on cloud marketplaces typically have programmed interfaces that allow business technologists, i.e. business users who are IT competent, to integrate the applications with existing IT systems (Oracle 2012). Expensive scarce IT professionals and consultants are typically not needed to install these applications, and if needed, the amount of time and effort is significantly reduced. New software should be ready for use after a few installation clicks. For example, GetFeedback (www.getfeedback.com) is a survey application listed on Salesforce AppExchange. It allows users to send surveys to customers, while the survey results will be autonomically synced into Salesforce CRM solution. Its main selling points, over other non-AppExchange survey applications, are ease of installation and a seamless integration capability with Salesforce so that any data received will be directly linked to the Salesforce CRM database.

The last benefit is cost reduction. Cloud marketplace customers can pay for software using a wide range of pricing models, e.g. per user, per month, per hour of usage and per amount of data ingested. These pricing models vary by cloud marketplace and software types. However, a 
basic principle remains constant: customers only pay for the portion of services that they use. For example, if there is only one person using the software for a given period of time, customers can subscribe to one seat for that period and no further. This contrasts with the old software licensing model, in which customers have to purchase a software license regardless of how much and how often they might use the software. In addition, software on cloud marketplaces usually offer a free trial period to allow customers to decide if the software is right for them (Phelan 2015). This reduces upfront costs for customers and provides cashflow advantages as firms scale up and down.

The next two sections present mini-case studies on two B2B cloud marketplaces. The first case is Salesforce AppExchange, a B2B cloud marketplace offering both applications and APIs, and the second is RapidAPI, a B2B API marketplace. Both mini-case studies focus on how customers could use B2B cloud marketplaces to generate, capture and measure business value.

\subsection{Mini-Case Study I: Salesforce AppExchange}

Salesforce AppExchange is a B2B cloud application and API marketplace for Salesforce customers, developers and partners. All applications listed on AppExchange are certified as compatible with Salesforce.com and preintegrated with Salesforce.com. There are thousands of applications available on the marketplace for a wide variety of use cases including sales, marketing, integration, customer service, manufacturing, analytics, and back office administration. Pricing models also vary including free, paid and discounted for non-profit.

Localytics (www.localytics.com) is a software vendor listed on Salesforce AppExchange. Localytics has been an App Innovation Partner with the Salesforce Marketing Cloud since 2016. Their software enables Salesforce.com and Localytics customers leverage mobile user data to create a closed loop system with regard to a customer across channels. Initially, Localytics' application combined the power of geofencing - the use of GPS or RFID technology to create a virtual geographic boundary to trigger a software to responsed when a mobile device is entering a particular areawith Salesforce.com. Firms can leverage Salesforce.com and Localytics to extract value from and generate new value for customers. For example, Priceline.com uses this combination to send targeted location-based marketing messages to its customers (Localytics 2019). These marketing 
messages are directed at customers who travel without any advance hotel and/or car-rental bookings. To capture these customers, the marketing messages focus on one-day special offers for hotels and car rentals. A combination of two powerful technologies, geofencing and CRM, with clever marketing messages captures business value for Priceline by allowing the firm to trigger a response to its customers' purchase decisions in real time. The business value from using the Localytics application can be conveniently measured in Salesforce.com through the conversion rate of customers who receive a message and make a booking within the same day, as well as the raw dollar amount received for the bookings. Today, Localytics is offered through AppExchange as an API for as little as US\$1 per customer per year. Salesforce.com, Localytics and their joint customers share from combinatory innovation, scale, convenience, and reduced costs.

\subsection{Mini-CASE STUdy II: RAPIDAPI}

RapidAPI is the largest API marketplace and is a merger between RapidAPI and the former Meshape API marketplace. Firms looking for standard APIs can subscribe to API services available on the marketplace to achieve their business purposes without reinventing the wheel. There are three pricing plans for APIs: free, freemium and paid. APIs are made available by categories such as storage, logistics, database, and search etc. In comparison to a traditional approach to build and maintain one's own APIs, using the RapidAPI marketplace allows firms to accelerate go-to-market, innovate, and generate business value much faster. API marketplaces allow firms to focus on what they do best, whether that is designing a cloud, web or mobile application to meet their target market. They can source relevant APIs through the RapidAPI marketplace and thereby significantly reduce an application/website development time cycle. The APIs are designed to meet the requirements of RapidAPI's marketplace including customer support; vendors can be removed from the marketplace for poor quality or service.

In the Irish Institute for Digital Business, we recently worked with a startup, which for the purposes of this case study, we shall call RecipeApp. The client had no technical background but extensive experience in hospitality. They wanted to build an application that provided (i) recipe data including ingredients, calories and portion sizes, and (ii) the ability to use this data for menu planning, budgeting and procurement, by small business such as delicatessens, coffee shops etc. Instead of investing in a significant effort, in terms of cost, time and human resources, to 
research and compile a database of recipes, ingredients and nutrition data, they were able to leverage the Recipe-Food-Nutrition API from Spoonacular available on the RapidAPI marketplace to provide access to over 365,000 healthy recipes, comprising over 2,600 ingredients, and 115,000 menu items. This API provided a wide range of data including, nutrition analysis, indicate cost breakdown, cooking tips, related recipes, scaling/converting, wine pairings and much more. Not only was the client able to access the data they needed but the additional data inspired additional features and functionality for RecipeApp. Furthermore, from the time of the decision to test Spoonacular, the API was implemented through RapidAPI within an hour at no cost. Indeed the first 150 API calls were free, ideal for testing, after which cost per call started at US $\$ 0.002$ per point up to 10,000 points per day. For a startup, this predictable and scalable pricing was critical. Using an off-the-shelf API through a cloud marketplace enabled the startup to acclerate their time to market, innovate and demonstrate their proof-of-concept in a fraction of the cost, time and effort if they were to undertake all the data collection or API development themselves.

\subsection{Conclusion}

This chapter explores B2B cloud marketplaces at both structural and functional levels. From a structural perspective, B2B cloud marketplaces can be classified as a type of CSB (Fowley et al. 2013). At a functional level, firms can generate and capture business value from such marketplaces in a variety of ways depending on their role in the marketplace, be it a marketplace operator, a software vendor, or a customer. While the public is very familiar with consumer app marketplaces like Apple AppStore or Google Play, the general public, business decision makers and indeed, scholars, may be less familiar with cloud marketplaces designed specifically for businesses. In particular, we highlight the emergence of API marketplaces both in their own right and as part of what was traditionally called cloud application marketplaces. We illustrate these with two mini-case studies on Salesforce AppExchange and RapidAPI. There is a paucity of business research in this area. As such, we encourage further research not only on business value research but the antecedents and consequences of participation in cloud marketplaces for different actors and sectors. Similarly, we call for business research on other cloud service brokerage tiers. 


\section{REFERENCES}

Achim, Monica-Violeta, Sorin-Nicolae Borlea, and Codruța Mare. 2016. Corporate Governance and Business Performance: Evidence for the Romanian Economy. Journal of Business Economics and Management 17 (3): 458-474.

Adabi, Sepideh, Mozhgan Mosadeghi, and Samaneh Yazdani. 2018. A Real-World Inspired Multi-strategy based Negotiating System for Cloud Service Market. Journal of Cloud Computing 7 (1): 17.

AppDirect. 2013. Putting the Cloud Within Reach. http://go.appdirect.com/ hs-fs/hub/390882/file-1311910432-pdf/Collateral/Whitepaper-_Putting_ the_Cloud_Within_Reach.pdf.

AWS. 2016. AWS Marketplace Product Support Connection Onboarding and Seller Guide. Accessed 15 January 2017. https://s3 amazonaws.com/awsmploadforms/AWS-Marketplace-Product-Support-Connection-Onboardingand-Seller-Guide.pdf.

Brokaw, Lisa. 2014. How to Win With a Multisided Platform Business Model. https://sloanreview.mit.edu/article/how-to-win-with-a-multisidedplatform-business-model/.

Cantara, Michele. 2015. Four Best Practices for Customization Brokerage of Business Processes in the Cloud. Accessed 27 April 2016. https://www.gartner.com/doc/3106718/best-practices-customization-brokerage-business.

Carraway, Debbie, Everette Allen, Charles Cline, Bill Coker, Leslie Dare, Danny D. Davis, Dan Deter et al. 2015. Adopting Cloud Services at NC State: Guidelines and Considerations. Accessed 22 December 2016. https://oit. ncsu.edu/campus-it/cloud-services-at-nc-state/.

Columbus, Louis. 2017. RightScale 2017 State of the Cloud Report: Azure Gaining in Enterprises. https://www.forbes.com/sites/louiscolumbus $/ 2017 / 02 / 18 /$ rightscale-2017-state-of-the-cloud-report-azure-gainingin-enterprises/\#415baf4b8481.

DOD. 2014. Department of Defense Cloud Computing Security Requirements Guide. Accessed 22 December 2016. http://iase.disa.mil/cloud_security/ Documents/u_cloud_srg_vlr0-36.pdf.

DPC. 2015. Data Security Guidance. https://www.dataprotection.ie/viewdoc. asp?DocID=1091. Accessed 30 September 2015.

Elhabbash, Abdessalam, Faiza Samreen, James Hadley, and Yehia Elkhatib. 2019. Cloud Brokerage: A Systematic Survey. ACM Computing Surveys (CSUR) 51 (6): 119.

Evans, David S., and Richard Schmalensee. 2005. The Industrial Organization of Markets with Two-Sided Platforms. National Bureau of Economic Research.

FedRAMP. 2019. Pursuing a FedRAMP Ready Designation. https://www. fedramp.gov/pursuing-a-fedramp-ready-designation/.

Fischer, Juli. 2012. MaaS (Marketplace-as-a-Service). Accessed 28 June 2016. http://info.appdirect.com/resources/knowledge/marketplace-as-a-service. 
Fowley, Frank, Claus Pahl, and Li Zhang. 2013. A Comparison Framework and Review of Service Brokerage Solutions for Cloud Architectures. Service-Oriented Computing-ICSOC 2013 Workshops. Springer.

Gill, Nivi, Sean Hogan, and Mike Harvath. 2016. How To Build Badass Cloud Companies-By Avoiding 10 Common Mistakes. Accessed 3 January 2017. https://www.salesforce.com/video/300361/.

Kortright, Scott. 2018. Benefits of Single Sign-on Solutions. http://blog.identityautomation.com/benefits-of-single-sign-on-solutions.

Lheureux, Benoit, Daryl C. Plummer, Neil Wynne, Bill Caffery, James D. Buckner, and Ian Baeazley. 2012. A CIO Primer on Cloud Services Brokerage. Accessed 27 April 2016. https://www.gartner.com/doc/2201415/ cio-primer-cloud-services-brokerage.

Localytics. 2019. priceline.com https://appexchange.salesforce.com/servlet/ servlet.FileDownload?file=00P3A00000a0r8BUAQ.

MacInnes, Billy. 2017. Working Out What Makes a Good Cloud Marketplace. https://www.computerweekly.com/microscope/news / 450420067 / Working-out-what-makes-a-good-cloud-marketplace.

Malhotra, Arvind, and Ann Majchrzak. 2012. How Virtual Teams Use Their Virtual Workspace to Coordinate Knowledge. ACM Transactions on Management Information Systems (TMIS) 3 (1): 6.

MarketsandMarkets. 2015. Cloud Services Brokerage Market by Types-Global Forecast to 2020. Accessed 15 February 2016. http://www.researchandmarkets.com/research/xwbvvz/cloud_services.

Mecca, Giansalvatore, Michele Santomauro, Donatello Santoro, and Enzo Veltri. 2016. On Federated Single Sign-On in e-Government Interoperability Frameworks. International Journal of Electronic Governance 8 (1): 6-21.

Microsoft Azure. 2016a. How Do I Choose a Cloud Service Provider?. Accessed 21 December 2016. https://azure.microsoft.com/en-us/overview/ choosing-a-cloud-service-provider/.

2016b. How to Publish and Manage an Offer in the Azure Marketplace. Accessed 2 January 2017. https://docs.microsoft.com/en-us/azure/ marketplace-publishing/marketplace-publishing-getting-started\#supportedtypes-of-solutions.

Morin, Jean-Henry, Jocelyn Aubert, and Benjamin Gateau. 2012. Towards Cloud Computing SLA Risk Management: Issues and Challenges. 2012 45th Hawaii International Conference on System Sciences. IEEE.

Oracle. 2012. Ten Questions to Ask Your Cloud Vendor Before Entering the Cloud. An Oracle White Paper. http://www.oracle.com/us/products/applications / 10-questions-for-cloud-vendors-1639601.pdf.

- 2015. Market Your Oracle Cloud Apps and Services. https://cloud.oracle.com/_downloads/eBook_Marketplace_res2/Oracle_Cloud_Marketplace_ Partner_Portal.pdf. 
Phelan, Gemma. 2015. Selling Cloud Services on the Digital Marketplace. https://digitalmarketplace.blog.gov.uk/2015/08/28/ selling-cloud-services-on-the-digital-marketplace/.

Salesforce. 2016. Salesforce Partner Program Guide for ISVs: A Detailed Guide to the ISV Program. Accessed 15 January 2017. https://partners.salesforce. com/s/ISVPartnerProgramGuidePY2017.pd.

Sen, Jaydip. 2015. Security and Privacy Issues in Cloud Computing. In Cloud Technology: Concepts, Methodologies, Tools, and Applications, 1585-1630. IGI Global.

Sill, Alan, Annie Sokol, Craig Lee, David Harper, Eugene Luster, Frederic de Vaulx, Gary Massaferro, and Gilbert Pilz. 2013. NIST Cloud Computing Standards Roadmap. NIST Special Publication 500-291, Version 2. Accessed 13 January 2016. http://www.nist.gov/itl/cloud/upload/NIST_ SP-500-291_Version-2_2013_June18_FINAL.pdf.

Open Access This chapter is licensed under the terms of the Creative Commons Attribution 4.0 International License (http://creativecommons.org/licenses/ by $/ 4.0 /$ ), which permits use, sharing, adaptation, distribution and reproduction in any medium or format, as long as you give appropriate credit to the original author(s) and the source, provide a link to the Creative Commons licence and indicate if changes were made.

The images or other third party material in this chapter are included in the chapter's Creative Commons licence, unless indicated otherwise in a credit line to the material. If material is not included in the chapter's Creative Commons licence and your intended use is not permitted by statutory regulation or exceeds the permitted use, you will need to obtain permission directly from the copyright holder.

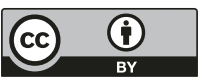

